\title{
Quantum Monte Carlo calculations of the dimerization energy of borane
}

\author{
Francesco Fracchia, Dario Bressanini, ${ }^{\text {a) }}$ and Gabriele Morosi ${ }^{\text {b) }}$ \\ Dipartimento di Scienze Chimiche e Ambientali, Universitá dell'Insubria, via Lucini 3, 22100 Como, Italy
}

(Received 17 June 2011; accepted 5 August 2011; published online 1 September 2011)

\begin{abstract}
Accurate thermodynamic data are required to improve the performance of chemical hydrides that are potential hydrogen storage materials. Boron compounds are among the most interesting candidates. However, different experimental measurements of the borane dimerization energy resulted in a rather wide range $(-34.3$ to -39.1$) \pm 2 \mathrm{kcal} / \mathrm{mol}$. Diffusion Monte Carlo (DMC) simulations usually recover more than $95 \%$ of the correlation energy, so energy differences rely less on error cancellation than other methods. DMC energies of $\mathrm{BH}_{3}, \mathrm{~B}_{2} \mathrm{H}_{6}, \mathrm{BH}_{3} \mathrm{CO}, \mathrm{CO}$, and $\mathrm{BH}_{2}{ }^{+}$allowed us to predict the borane dimerization energy, both via the direct process and indirect processes such as the dissociation of $\mathrm{BH}_{3} \mathrm{CO}$. Our $\mathrm{D}_{\mathrm{e}}=-43.12(8) \mathrm{kcal} / \mathrm{mol}$, corrected for the zero point energy evaluated by considering the anharmonic contributions, results in a borane dimerization energy of $-36.59(8) \mathrm{kcal} / \mathrm{mol}$. The process via the dissociation of $\mathrm{BH}_{3} \mathrm{CO}$ gives $-34.5(2) \mathrm{kcal} / \mathrm{mol}$. Overall, our values suggest a slightly less $\mathrm{D}_{\mathrm{e}}$ than the most recent $\mathrm{W} 4$ estimate $\mathrm{D}_{\mathrm{e}}=-44.47 \mathrm{kcal} / \mathrm{mol}$ [A. Karton and J. M. L. Martin, J. Phys. Chem. A 111, 5936 (2007)]. Our results show that reliable thermochemical data for boranes can be predicted by fixed node (FN)-DMC calculations. () 2011 American Institute of Physics. [doi:10.1063/1.3629778]
\end{abstract}

\section{INTRODUCTION}

Chemical hydrides are potential hydrogen storage materials. If hydrogen is bound to lightweight elements such as boron and nitrogen, materials with high hydrogen content per unit weight can be obtained. ${ }^{1}$ Accurate thermodynamic data are required to improve their performance, to optimize both the release process and the regeneration system. When the thermodynamic properties are not known, or their accuracy is not good enough, theoretical values can be obtained by a variety of methods. The field of computational thermochemistry has been reviewed by Martin $^{2}$ and more recently by van Speybroeck et al. ${ }^{3}$ DFT calculations are computationally cheap, but their results depend on the exchange-correlation functional employed and on the basis set. Ab initio methods require the extrapolation to the complete basis set and the full CI limits and so they are limited to very small systems. Quantum Monte Carlo methods are an appealing alternative to standard quantum chemistry approaches. Very accurate results can be calculated by diffusion Monte Carlo (DMC) simulations, usually in the fixed node approximation (FN-DMC), the nodal error being the only obstacle to the exact solution of the Schrödinger equation. Benchmark results ${ }^{4}$ on the G2 set of 55 molecules show that on average $95 \%$ of the correlation energy can be recovered. Those calculations used a single Slater-Jastrow trial wave function. The molecular orbitals were a linear combination of Slater-type orbitals of QZ4P quality. Unfortunately, the error cancellation when computing energy differences, such as bond energies and chemical reaction energies, is not systematic, resulting in bond energies with a mean absolute deviation of $3.2 \mathrm{kcal} / \mathrm{mol}$ from the experimental reference values. Better results can be obtained

\footnotetext{
a)Electronic mail: Dario.Bressanini@uninsubria.it.

${ }^{b)}$ Electronic mail: Gabriele.Morosi@uninsubria.it.
}

improving the nodal surfaces by multideterminantal wave functions ${ }^{5}$ and further by backflow functions. ${ }^{6}$ However, not all the determinants that can be added to the reference wave function improve the nodal surface. The selection of favorable determinants is still a matter of trial and error, even if previous experiences might suggest some hints. When applied to systems with few electrons, the DMC method needs more computer time than standard ab initio methods. However, it scales favorably with the number of electrons and can be easily implemented on massively parallel computers. The goal of this work is to investigate the potential of DMC method for thermochemistry, simulating several boron hydrides.

\section{A SHORT HISTORICAL OVERVIEW ON BORANES}

Boranes are a family of molecules that for many years, since their experimental discovery due to Alfred Stock in the first decades of the last century, ${ }^{7}$ puzzled chemists.

The first attempt to elucidate the structure of diborane $\left(\mathrm{B}_{2} \mathrm{H}_{6}\right)$ dates back to 1925 (Ref. 8) using the new x-ray diffraction technique. At that time diborane was thought to have a structure similar to that of ethane. This interpretation was later supported by Bauer in 1937 using electron diffraction techniques. ${ }^{9}$ However, this type of structure was not supported by the prevailing valence bond theory, since it would require 14 electrons, shared by the various bonds, and not only 12 . The fact that diborane is diamagnetic was already established in 1934 (Ref. 10) and this means that there are no unpaired electrons. Many different alternative valence bond structures were suggested, including resonant forms between molecules with single electron bonds or resonance between ionic structures involving $\mathrm{BH}_{4}{ }^{-}$and $\mathrm{BH}_{2}{ }^{+}$. Among different possibilities, Schlesinger and Burg ${ }^{11}$ in 1942 listed the structure, with hydrogen in bridge position between the two 
TABLE I. Experimental borane dimerization energies.

\begin{tabular}{lcc}
\hline \hline Energy $(\mathrm{kcal} / \mathrm{mol})$ & Year & References \\
\hline-38.3 & 1964 & Garabedian and Benson (Ref. 22) \\
$-37.1 \pm 4$ & 1965 & Fehlner and Koski (Ref. 23) \\
-35.0 & 1966 & Burg and Fu (Ref. 24) \\
-39 & 1964 & Fehlner and Koski (Ref. 25) \\
$-55 \pm 8$ & 1964 & Sinke et al. (Ref. 26) \\
-59 & 1967 & Wilson and McGee (Ref. 27) \\
-59 & 1969 & Ganguli and McGee (Ref. 28) \\
$-36 \pm 3$ & 1969 & Fehlner and Mappes (Ref. 29) \\
$(-34.3$ to -39.1$) \pm 2$ & 1988 & Ruscic et al. (Ref. 30) \\
\hline \hline
\end{tabular}

boron atoms, that we now know is the correct one. In 1943 Longuet-Higgins and Bell ${ }^{12}$ reviewed all the experimental data available at that time and concluded that the structure with the bridged hydrogens was the most likely. Around the same time a few computational investigations, most notably by Mulliken, ${ }^{13}$ Pitzer, ${ }^{14}$ and Walsh, ${ }^{15}$ using molecular orbital theory and valence bond theory, gave theoretical support to that structure. In 1949 Longuet-Higgins ${ }^{16}$ proposed the famous two-electron three-center bond. Further investigations in the following decade, most notably by Lipscomb,${ }^{17}$ finally led to the elucidation of the electronic structure of diborane and higher boranes. Lipscom was awarded the Nobel Prize in 1976 "for his studies on the structure of boranes illuminating problems of chemical bonding". 18

\section{A. The experimental estimation of the dimerization energy of borane}

The experimental determination of the dimerization energy of borane $\left(2 \mathrm{BH}_{3} \rightarrow \mathrm{B}_{2} \mathrm{H}_{6}\right)$ is not an easy task since borane is extremely reactive. Table I reviews most of the experimental values. A direct calorimetric measure has not been obtained so far due to the fact that the activation energy of the reaction is zero. ${ }^{19}$ Pauling $^{20}$ was one of the firsts to estimate its value at approximately $-23.9 \mathrm{kcal} / \mathrm{mol}$ based on considerations on the electronegativity of boron and hydrogen. Early indirect measures of the dimerization energy were based on kinetic studies of the reaction of diborane with carbon monoxide $\mathrm{B}_{2} \mathrm{H}_{6}+2 \mathrm{CO}=2 \mathrm{BH}_{3} \mathrm{CO}:^{21}$ the dimerization energy can be estimated from the knowledge of the dissociation energy of carbonyl borane, $\leq 23.73 \mathrm{kcal} / \mathrm{mol}$ according to Garabedian and Benson ${ }^{22}$ and $23.1 \pm 2 \mathrm{kcal} / \mathrm{mol}$ according to Fehlner and Koski. ${ }^{23}$

The dissociation energy of $\mathrm{BH}_{3} \mathrm{CO}$ can be evaluated using the relation $\mathrm{D}\left(\mathrm{BH}_{3} \mathrm{CO}\right)=\mathrm{E}_{\mathrm{a}}\left(\mathrm{BH}_{3} \mathrm{CO} \rightarrow \mathrm{BH}_{3}+\mathrm{CO}\right)$ $-\mathrm{E}_{\mathrm{a}}\left(\mathrm{BH}_{3}+\mathrm{CO} \rightarrow \mathrm{BH}_{3} \mathrm{CO}\right)$, measuring the activation energies $\mathrm{E}_{\mathrm{a}}$ of the dissociation reaction and assuming that the activation energy of the formation reaction is zero. We have confirmed this hypothesis by inspection of the potential energy surface of the reaction $\mathrm{BH}_{3}+\mathrm{CO} \rightarrow \mathrm{BH}_{3} \mathrm{CO}$ at more than 200 fixed B-C distances: calculations were performed at B3LYP level using an aug-cc-pVTZ basis set. ${ }^{31}$ The dimerization energy of borane was also estimated, during the 1960s, using electron impact ionization mass spectroscopy. Various experiments $^{26-28}$ tried to measure the energies of the processes

$$
\begin{aligned}
& \mathrm{BH}_{3}+h v \rightarrow \mathrm{BH}_{3}^{+}+e^{-} \\
& \mathrm{B}_{2} \mathrm{H}_{6}+h v \rightarrow \mathrm{BH}_{3}^{+}+\mathrm{BH}_{3}+e^{-}
\end{aligned}
$$

from which the dimerization energy of diborane can be obtained by difference. However, the results did not agree with those from the kinetic studies, as can be seen from Table I: the values were significantly higher.

Ruscic et al..$^{30}$ in 1988 reviewed those earlier mass spectroscopic estimations and pointed out some flaws in their assumptions. In particular the borane cation $\mathrm{BH}_{3}^{+}$observed in the experiments could also come from the fragmentation of $\mathrm{B}_{2} \mathrm{H}_{5}^{+}$, a higher energy process.

They proceeded then, using a variety of experimental data, to give a reliable estimate of the dimerization energy: $(-34.3$ to -39.1$) \pm 2 \mathrm{kcal} / \mathrm{mol}$. To our knowledge, this is the most recent experimental estimation of this quantity.

\section{B. Ab initio calculations}

A lot of theoretical work has been devoted to the computation of the dimerization energy of borane, as can be seen from Table II. Early calculations were based on the simple Hartree-Fock method using small basis sets; they predicted smaller dimerization energies than what might have been expected from the experimental results. As the computational power increased over the years, combined with the use of more sophisticated methods and larger basis sets, the computed values grew more in agreement with the experiment. However, there is not yet a consensus on what is the exact value of the dimerization energy of borane, the calculations of Feller et al. ${ }^{32}$ and Karton and Martin ${ }^{33}$ probably being, at the moment, the most accurate.

\section{METHODOLOGY AND WAVE FUNCTION}

We performed fixed node diffusion Monte Carlo calculations on $\mathrm{BH}_{3}, \mathrm{~B}_{2} \mathrm{H}_{6}, \mathrm{BH}_{3} \mathrm{CO}, \mathrm{CO}$, and $\mathrm{BH}_{2}{ }^{+}$. In all cases we employed both single determinant trial wave functions and short expansions of configuration state functions times a Jastrow factor. The Jastrow factor was optimized by the variance minimization method. ${ }^{42}$

FN-DMC simulations were performed for borane at the equilibrium geometry computed by Schuurman et al. $^{43}$ and for diborane at the geometry determined by Duncan and Harper. ${ }^{44}$ Several basis sets of increasing size were confronted: for $\mathrm{B}_{2} \mathrm{H}_{6}$ the gain was 4.5 mhartree on going from DZ to TZ basis set, 0.7 mhartree from TZ to QZ, and 0.2 mhartree from QZ to Cade-Huo basis set. ${ }^{45}$ This basis set gave the best results at DMC level for a single determinant trial wave functions. Using this basis set we generated both $\mathrm{HF}$ and B3LYP molecular orbitals and tested them in FN-DMC simulations. We found that, systematically, B3LYP orbitals give lower FN-DMC energies (for example, 1.9 and 0.5 mhartree are recovered for $\mathrm{B}_{2} \mathrm{H}_{6}$ and $\mathrm{BH}_{3}$, respectively, on going from HF to B3LYP orbitals). Several papers have appeared in the last few years investigating the nodal structure of exact and 
TABLE II. Calculated borane dimerization energy ( $\mathrm{kcal} / \mathrm{mol})$.

\begin{tabular}{|c|c|c|c|c|c|c|}
\hline Year & Method & Basis set & $\Delta \mathrm{E}_{\mathrm{e}}$ & $\Delta \mathrm{E}_{0}$ & $\mathrm{ZPE}$ & References \\
\hline 1973 & $\mathrm{HF}$ & DZ & -11.5 & & & Edmiston and Lindner (Ref. 34) \\
\hline 1974 & $\mathrm{HF}$ & $\mathrm{TZV}+$ & -19 & & & Marynick et al. (Ref. 35) \\
\hline 1974 & $\mathrm{HF}$ & $\mathrm{TZV}+$ & -20.7 & & & Ahlrichs (Ref. 36) \\
\hline 1974 & IEPA & $\mathrm{TZV}+$ & -44.3 & & & Ahlrichs (Ref. 36) \\
\hline 1974 & CEPA & $\mathrm{TZV}+$ & -36.6 & & & Ahlrichs (Ref. 36) \\
\hline 1979 & SDQ-MBPT & DZ & -35.2 & -30.2 & 5.0 & Redmon et al. (Ref. 37) \\
\hline 1987 & $\mathrm{HF}$ & $6-311 \mathrm{G}++$ & -21.9 & & & Page et al. (Ref. 38) \\
\hline 1987 & MP2 & $6-311 \mathrm{G}++$ & -43.99 & -37.89 & 6.1 & Page et al. (Ref. 38) \\
\hline 1987 & MP4 & $6-311 \mathrm{G}++$ & -43.14 & -37.0 & 6.1 & Page et al. (Ref. 38) \\
\hline 1988 & G1 & & -42.6 & -36.2 & 6.4 & Curtiss and Pople (Ref. 39) \\
\hline 1992 & CCSD & DZP & -36.5 & -30.0 & 6.5 & Shen and Schaefer (Ref. 40) \\
\hline 1994 & B3LYP & TZ2P & -39.3 & -32.6 & 6.7 & Barone et al. (Ref. 41) \\
\hline 1998 & $\operatorname{CCSD}(\mathrm{T})$ & CBS & -44.3 & -38.2 & 6.2 & Feller et al. (Ref. 32) \\
\hline \multirow[t]{3}{*}{2007} & W4 & & -44.47 & -37.9 & 6.53 & Karton and Martin (Ref. 33) \\
\hline & FN-DMC & & $-43.12(8)$ & $-36.59(8)$ & 6.53 & Present work \\
\hline & FN-DMC via $\mathrm{D}\left(\mathrm{BH}_{3} \mathrm{CO}\right)$ & & & $-34.5(2)$ & & Present work \\
\hline
\end{tabular}

approximate trial wave functions, 5,46 but a general understanding is still lacking and we do not have a solid mathematical argument why B3LYP orbitals should generate better many body fermion nodes, or if this is a general feature. At present we can only take this as an empirical observation on many different systems, to the point to suggest to use exchange-correlation functionals with variable exact-exchange component as a method to minimize the nodal error. ${ }^{47}$ Given the importance of the nodal structure on the FN-DMC simulations, it should be worth pursuing the investigation on which single-particle method gives better many-body nodes and why. The differences between the FN-DMC energies computed using the HF or B3LYP orbitals decrease as the basis quality increases, but, again, the reasons why this happens are not clear at the moment.

On the basis of these results all the calculations reported in this paper have been performed using B3LYP orbitals. For borane and diborane several multideterminant wave functions were tested, selecting the configurations with highest CI coefficients and optimizing them: ${ }^{48}$ very small energy decreases were obtained including for borane excitations from $\sigma$ to $\pi$ orbitals and for diborane excitations to the first $\mathrm{B}_{2 \mathrm{~g}}$ orbital. The energy gain was 0.4 mhartree for borane and 0.2 mhartree for diborane. As a comparison, the boron atom FN-DMC energy computed with a single determinant trial wave function is 15 mhartree higher than our best value (-24.65311(5) hartree) computed with a fairly long multideterminant expansion (115 determinants). This result recovers $99.4 \%$ of the correlation energy. Open shell systems require many determinants to define fairly good nodal surfaces, while closed shell single determinant trial wave functions give already accurate nodal surfaces and their improvement is rather a difficult task. In conclusion we expect our borane and diborane energies to recover a substantial percentage of correlation energy. We have defined a compact trial wave function selecting by hand those determinants that gave a non-negligible lowering in the FN-DMC energy. The hard work required for this selection process might however help to get some hints on the factors that contribute to improve the nodal surface, which is still an open problem. For example, we found that excitations to orbitals with a different symmetry from the orbitals of the reference configuration seem important, a result that reminds the importance of configurations different than the ground one for atomic wave functions. A different approach has been proposed by several groups, ${ }^{49-51}$ based on the full optimization at variational Monte Carlo (VMC) level of the trial wave functions. These functions give lower VMC and FN-DMC energies than those built on a fixed basis set (either HF or DFT or natural orbitals basis sets) and it seems possible, using a large set of determinants, to systematically improve the quality of the wave function. Due to our determinant selection scheme our VMC energies are not necessarily a measure of the quality of the nodal surfaces. Another approach aimed at accurate calculations of dissociation energies by compact trial wave functions proposed by Braïda et al.: ${ }^{52}$ they used a valence bond formulation for the determinantal part of the trial wave function, a way to reduce the number of determinants required to describe the static correlation with respect to the molecular orbital theory.

For carbon and oxygen we employed the basis set optimized by Cade and $\mathrm{Huo}^{45}$ for the corresponding hydrides. For $\mathrm{CO}$ at the equilibrium geometry ${ }^{53}$ the energy calculated by B3LYP orbitals can be slightly improved (5 mhartree) using 11 determinants including excitations from $\sigma$ to $\pi^{*}$ and from $\pi$ to $\pi^{*}$ orbitals. For this molecule a value of -113.326 hartree for the nonrelativistic, nonvibrating, infinite mass nuclei total energy has been derived. ${ }^{54}$ Together with the Hartree-Fock limit -112.7909 hartree, it allows us to evaluate that our FN-DMC energy -113.2993(3) hartree recovers $95 \%$ of the correlation energy. Having defined the trial wave functions for the other molecules with the same criteria we are confident that all our energies should have the same accuracy. For $\mathrm{BH}_{3} \mathrm{CO}$ at the equilibrium geometry ${ }^{55}$ the expansion of trial wave function is similar to the one of $\mathrm{CO}$. Molecular FN-DMC energies are shown in Table III together with zero point energies (ZPE) taken from literature or computed by a quartic force field ${ }^{56}$ for $\mathrm{BH}_{3} \mathrm{CO}$ at MP2/aug-cc-pVTZ level and for $\mathrm{BH}_{2}{ }^{+}$at $\mathrm{CCSD}(\mathrm{T}) / \mathrm{cc}-\mathrm{pVTZ}$ level. 
TABLE III. Fixed-node diffusion Monte Carlo energies (hartree).

\begin{tabular}{lccc}
\hline \hline Molecule & FN-DMC energy & ZPE & $\mathrm{E}_{0}$ \\
\hline $\mathrm{BH}_{3}$ & $-26.59828(6)$ & $0.02607^{\mathrm{a}}$ & $-26.57221(6)$ \\
$\mathrm{B}_{2} \mathrm{H}_{6}$ & $-53.2653(1)$ & $0.06255^{\mathrm{a}}$ & $-53.2028(1)$ \\
$\mathrm{CO}$ & $-113.2993(3)$ & $0.00493^{\mathrm{b}}$ & $-113.2944(3)$ \\
$\mathrm{BH}_{3} \mathrm{CO}$ & $-139.9374(2)$ & 0.03658 & $-139.9008(2)$ \\
$\mathrm{BH}_{2}{ }^{+}$ & $-25.62249(6)$ & 0.01719 & $-25.60530(6)$ \\
\hline \hline
\end{tabular}

${ }^{\mathrm{a}}$ Reference 33 .

${ }^{\mathrm{b}}$ Reference 57.

\section{RESULTS}

From Table III the borane dimerization energy is $\Delta \mathrm{E}_{\mathrm{e}}$ $=-0.0687(1)$ hartree $=-43.12(8) \mathrm{kcal} / \mathrm{mol}$. The $\mathrm{BH}_{3}$ zero point energy $16.36 \mathrm{kcal} / \mathrm{mol}=0.02607$ hartree computed by Karton and Martin ${ }^{33}$ has been slightly corrected by Meier et al. ${ }^{58}$ : their value for ${ }^{11} \mathrm{BH}_{3}$ is $5715.5 \mathrm{~cm}^{-1}$, that is 0.02604 hartree. However, to correct the borane dimerization energy for the zero point energy we used the value by Karton and Martin as computed with the same model as the diborane ZPE. In any case the difference is within one standard deviation from our FN-DMC energy. Our value is $\Delta \mathrm{E}_{0}$ $=-36.59(8) \mathrm{kcal} / \mathrm{mol}$, in good agreement with the best current experimental value $(-34.3$ to -39.1$) \pm 2 \mathrm{kcal} / \mathrm{mol}{ }^{30}$ Our results are shown in Table II. How do the trial wave functions used to compute the FN-DMC energies influence the dimerization energy? Using single determinant trial wave functions both for $\mathrm{BH}_{3}$ and $\mathrm{B}_{2} \mathrm{H}_{6}$ the dimerization energy is $-36.8(1) \mathrm{kcal} / \mathrm{mol}$. HF orbitals, as we already said, gave worse energies than B3LYP orbitals, of the order of 12 mhartree, but again the dimerization energy is $-36.8(1)$ $\mathrm{kcal} / \mathrm{mol}$. A slightly smaller basis set (VB1), ${ }^{59}$ that worsen the FN-DMC energy roughly 0.1 and 0.6 mhartree for $\mathrm{BH}_{3}$ and $\mathrm{B}_{2} \mathrm{H}_{6}$, respectively, again results in a dimerization energy of $36.7(1) \mathrm{kcal} / \mathrm{mol}$. These values are in statistical agreement with the value computed by our best results of Table II, indicating cancellation of the nodal error. Due to the wave function construction scheme we employed, we did not expect a substantial cancellation of error occurring at the VMC level and for this reason we did not include VMC energies in Tables III and IV.

From the thermodynamic cycle

$$
\begin{array}{ll}
2 \mathrm{BH}_{3} \mathrm{CO} \rightarrow \mathrm{B}_{2} \mathrm{H}_{6}+2 \mathrm{CO} & \Delta \mathrm{H}_{1}, \\
2 \mathrm{BH}_{3}+2 \mathrm{CO} \rightarrow 2 \mathrm{BH}_{3} \mathrm{CO} & \Delta \mathrm{H}_{2},
\end{array}
$$

TABLE IV. $\mathrm{BH}_{2}{ }^{+}$energy.

\begin{tabular}{llc}
\hline \hline Method & Energy (hartree) & References \\
\hline MP2/6-311G(d,p) & -25.5526 & DePuy et al. (Ref. 62) \\
QCISD(T)/cc-pVTZ & -25.5651 & DePuy et al. (Ref. 62) \\
G2 & -25.5568 & DePuy et al. (Ref. 62) \\
CBS-Q & -25.5543 & DePuy et al. (Ref. 62) \\
CCSD(T)/cc-pVTZ & -25.56494 & Zeng and Davico (Ref. 63) \\
FN-DMC & $-25.62249(6)$ & This work \\
Experimental estimate & -25.6227 & \\
\hline \hline
\end{tabular}

it is possible to obtain the borane dimerization energy. In a study of the mechanism of decomposition of $\mathrm{BH}_{3} \mathrm{CO}$ with formation of $\mathrm{B}_{2} \mathrm{H}_{6}$, Burg ${ }^{60}$ measured a value $9.142 \mathrm{kcal} / \mathrm{mol}$ for $\Delta \mathrm{H}_{2}$ at $326 \mathrm{~K}$, unfortunately, no estimate of the experimental uncertainty of this value was reported. The proposed relation between the dissociation energies of $\mathrm{BH}_{3} \mathrm{CO}$ and $\mathrm{B}_{2} \mathrm{H}_{6}$ was $\mathrm{D}\left(\mathrm{B}_{2} \mathrm{H}_{6}\right)=2 \mathrm{D}\left(\mathrm{BH}_{3} \mathrm{CO}\right)-9.142 \mathrm{kcal} / \mathrm{mol}$ at $326 \mathrm{~K}$. Our dissociation energy of $\mathrm{BH}_{3} \mathrm{CO} \rightarrow \mathrm{BH}_{3}+\mathrm{CO}$ is $\mathrm{D}_{\mathrm{e}}$ $=25.0(2) \mathrm{kcal} / \mathrm{mol}, \mathrm{D}_{0}(0 \mathrm{~K})=21.5(2) \mathrm{kcal} / \mathrm{mol}$ after $\mathrm{ZPE}$ correction at CCSD(T)/aug-cc-pVTZ level. Bauschlicher and Ricca ${ }^{61}$ computed $\mathrm{D}_{0}(0 \mathrm{~K})=21.1 \mathrm{kcal} / \mathrm{mol}$ at $\mathrm{CCSD}(\mathrm{T}) / \mathrm{aug}-$ cc-pVTZ level. Both theoretical values are smaller than the experimental upper limit of $23.73 \mathrm{kcal} / \mathrm{mol}$ found by Garabedian and Benson ${ }^{22}$ and are within one standard deviation from the value of $23.1 \pm 2 \mathrm{kcal} / \mathrm{mol}$ by Fehlner and Koski. ${ }^{23}$ Our result, corrected for $\mathrm{T}=326 \mathrm{~K}$, is $\mathrm{D}=22.9(2) \mathrm{kcal} / \mathrm{mol}$. Using the empirical relation found by Burg it is possible to give an independent estimate of the dimerization energy of diborane of $-34.5(2) \mathrm{kcal} / \mathrm{mol}$ (see Table II). This value is smaller than the direct estimate of the dimerization energy and near the lower bound of the experimental range.

Ruscic et al. ${ }^{30}$ estimated the dimerization energy of diborane by the following ionization processes:

$$
\begin{aligned}
& \mathrm{B}_{2} \mathrm{H}_{6}+h v \rightarrow \mathrm{BH}_{2}^{+}+\mathrm{BH}_{3}+\mathrm{H}+e^{-} \\
& \mathrm{BH}_{3}+h v \rightarrow \mathrm{BH}_{2}^{+}+\mathrm{H}+e^{-}
\end{aligned}
$$

giving an upper bound energy to the first process, at $0 \mathrm{~K}$, of $342.2(2) \mathrm{kcal} / \mathrm{mol}$ and of $295.6(5) \mathrm{kcal} / \mathrm{mol}$ to the second. Subtracting the two energies an estimate of $46.6(5) \mathrm{kcal} / \mathrm{mol}$ was obtained, a value much larger than the most likely estimate. They justified this overestimation by arguing that the first process goes through an intermediate ionization step, producing $\mathrm{B}_{2} \mathrm{H}_{5}^{+}$that subsequently fragments into $\mathrm{BH}_{2}^{+}$and $\mathrm{BH}_{3}$. The second process instead, they claimed, is a single stadium process and so the measured energy is the relevant one for that fragmentation.

In order to investigate the matter we performed a FNDMC simulation of $\mathrm{BH}_{2}^{+}$using a multideterminant trial wave function including $\sigma \rightarrow \pi$ excitations, built from B3LYP orbitals as already discussed above. $\mathrm{BH}_{2}^{+}$is a linear molecule whose bond length is not experimentally known. We optimized the geometry using CCSD(T)/cc-pV5Z obtaining a bond length of $1.167 \AA$. To our knowledge there is no accurate estimation of the total energy of this molecule available in the literature. Our FN-DMC result, along with other computed values available in the literature, is reported in Table IV. The experimental estimate -25.6227 hartree was computed from atomic non-relativistic energies, ZPE calculated at the $\operatorname{CCSD}(\mathrm{T}) / \mathrm{cc}-\mathrm{pVTZ}$ level of theory, $\mathrm{BH}_{3}$ atomization energy, and the experimental value for the process $\mathrm{BH}_{3}+h v \rightarrow \mathrm{BH}_{2}^{+}+\mathrm{H}+\mathrm{e}^{-}$.

The FN-DMC value largely improves the previous $a b$ initio results, and it is close to the experimental estimate.

Using our FN-DMC energy of $\mathrm{BH}_{3}$ reported previously, we compute the energy of the process $\mathrm{BH}_{3}+h v \rightarrow \mathrm{BH}_{2}^{+}+\mathrm{H}+\mathrm{e}^{-}$to be 292.99(5) $\mathrm{kcal} / \mathrm{mol}$, $2.61 \mathrm{kcal} / \mathrm{mol}$ lower than the upper limit measured by Ruscic et $a{ }^{30}{ }^{30}$ Anyway, this result supports their assumption that 
this reaction is a direct process. For the other process, $\mathrm{B}_{2} \mathrm{H}_{6}+h v \rightarrow \mathrm{BH}_{2}^{+}+\mathrm{BH}_{3}+\mathrm{H}+\mathrm{e}^{-}$we estimate an energy of 329.61(9) $\mathrm{kcal} / \mathrm{mol}, 12.6(4) \mathrm{kcal} / \mathrm{mol}$ lower than the upper bound $342.2(4) \mathrm{kcal} / \mathrm{mol}$ measured by Ruscic. Again this result supports their conjecture that what they measured was only an upper bound to the energy of the fragmentation process that presumably involves additional steps.

\section{CONCLUSIONS}

In this work we have evaluated the borane dimerization energy by $\mathrm{FN}-\mathrm{DMC}$ simulations of $\mathrm{BH}_{3}, \mathrm{~B}_{2} \mathrm{H}_{6}, \mathrm{BH}_{3} \mathrm{CO}, \mathrm{CO}$, and $\mathrm{BH}_{2}{ }^{+}$. These compounds enter in different processes that have been investigated to measure the dimerization energy. FN-DMC usually recovers some $95 \%$ of the correlation energy without resorting to basis extrapolations or to empirical post-HF methods such as G2MP2 or W4. For CO we have verified that our result recovers $95 \%$ of the correlation energy; having computed the other molecules with similar atomic and molecular basis sets makes us confident that their results should be in the same range of accuracy. FN-DMC values are variational and are affected only by the nodal error; we have empirically observed a nodal error cancellation comparing the values obtained by single and multideterminant trial wave functions. Our value for the $2 \mathrm{BH}_{3} \rightarrow \mathrm{B}_{2} \mathrm{H}_{6}$ process $(-36.59(8) \mathrm{kcal} / \mathrm{mol})$ is within the range of the experimental data and slightly less than the most recent theoretical results. ${ }^{32,33}$ In conclusion reliable thermochemical data can be predicted by FN-DMC calculations.

\section{ACKNOWLEDGMENTS}

This work has been done in the framework of a project financed by the University of Insubria.

${ }^{1}$ F. H. Stephens, V. Pons, and R. T. Baker, Dalton Trans. 2007, 2613.

${ }^{2}$ J. M. L. Martin, Annu. Rep. Comput. Chem. 1, 31 (2005).

${ }^{3}$ V. van Speybroeck, R. Gani, and R. J. Meier, Chem. Soc. Rev. 39, 1764 (2010).

${ }^{4}$ N. Nemec, M. D. Towler, and R. J. Needs, J. Chem. Phys. 132, 034111 (2010).

${ }^{5}$ D. Bressanini, G. Morosi, and S. Tarasco, J. Chem. Phys. 123, 204109 (2005).

${ }^{6}$ P. López Ríos, A. Ma, N. D. Drummond, M. D. Towler, and R. J. Needs, Phys. Rev. E 74, 066701 (2006).

${ }^{7}$ A. Stock, The Hydrides of Silicon and Boron (Cornell University, Ithaca, 1933).

${ }^{8}$ H. Mark and E. Pohland, Z. Kristallogr. 62, 103 (1925).

${ }^{9}$ S. H. Bauer, J. Am. Chem. Soc. 59, 1096 (1937).

${ }^{10}$ L. Farkas and H. Sachsee, Trans. Faraday Soc. 30, 331 (1934).

${ }^{11}$ H. I. Schlesinger and A. B. Burg, Chem. Rev. 31, 1 (1942).

${ }^{12}$ H. Longuet-Higgins and R. Bell, J. Chem. Soc 250 (1943).

${ }^{13}$ R. S. Mulliken, Chem. Rev. 41, 207 (1947).

${ }^{14}$ K. S. Pitzer, J. Am. Chem. Soc. 67, 1126 (1945).

${ }^{15}$ A. D. Walsh, J. Chem. Soc. 1947, 89.

${ }^{16}$ H. C. Longuet-Higgins, J. Chim. Phys. Phys.-Chim Biol. 46, 268 (1949).

${ }^{17}$ W. N. Lipscomb, Boron Hydrides (W. A. Benjamin Inc., New York, 1963).
${ }^{18}$ Nobel Lectures in Chemistry 1971-1980, edited by S. Forsén (World Scientific Publishing Co., Singapore, 1993).

${ }^{19}$ G. W. Mappes, S. A. Fridmann, and T. P. Fehlner, J. Phys. Chem. 74, 3307 (1970).

${ }^{20}$ L. Pauling, The Nature of the Chemical Bond and the Structure of Molecules and Crystals: An Introduction to Modern Structural Chemistry., 3rd ed. (Cornell University, Ithaca, 1960).

${ }^{21}$ A. B. Burg and H. I. Schlesinger, J. Am. Chem. Soc. 59, 780 (1937).

${ }^{22}$ M. E. Garabedian and S. W. Benson, J. Am. Chem. Soc. 86, 176 (1964).

${ }^{23}$ T. P. Fehlner and W. S. Koski, J. Am. Chem. Soc. 87, 409 (1965).

${ }^{24}$ A. B. Burg and Y. C. Fu, J. Am. Chem. Soc. 88, 1147 (1966).

${ }^{25}$ T. P. Fehlner and W. S. Koski, J. Am. Chem. Soc. 86, 2733 (1964).

${ }^{26}$ E. J. Sinke, J. G. A. Pressley, A. B. Baylis, and F. E. Stafford, J. Chem. Phys. 41, 2207 (1964).

${ }^{27}$ J. H. Wilson and J. H. A. McGee, J. Chem. Phys. 46, 1444 (1967).

${ }^{28}$ P. S. Ganguli and J. H. A. McGee, J. Chem. Phys. 50, 4658 (1969).

${ }^{29}$ T. P. Fehlner and G. W. Mappes, J. Phys. Chem. 73, 873 (1969).

${ }^{30}$ B. Ruscic, C. A. Mayhew, and J. Berkowitz, J. Chem. Phys. 88, 5580 (1988).

${ }^{31}$ F. Fracchia, Laurea Thesis, University of Insubria (2009).

${ }^{32}$ D. Feller, D. A. Dixon, and K. A. Peterson, J. Phys. Chem. A 102, 7053 (1998).

${ }^{33}$ A. Karton and J. M. L. Martin, J. Phys. Chem. A 111, 5936 (2007).

${ }^{34}$ C. Edmiston and P. Lindner, Int. J. Quantum Chem. 7, 309 (1973).

${ }^{35}$ D. S. Marynick, J. H. Hall, and W. N. Lipscomb, J. Chem. Phys. 61, 5460 (1974).

${ }^{36}$ R. Ahlrichs, Theor. Chem. Acc. 35, 59 (1974).

${ }^{37}$ L. T. Redmon, G. D. Purvis, and R. J. Bartlett, J. Am. Chem. Soc. 101, 2856 (1979).

${ }^{38}$ M. Page, G. F. Adams, J. S. Binkley, and C. F. Melius, J. Phys. Chem. 91, 2675 (1987).

${ }^{39}$ L. A. Curtiss and J. A. Pople, J. Chem. Phys. 89, 4875 (1988).

${ }^{40}$ M. Z. Shen and H. F. Schaefer, J. Chem. Phys. 96, 2868 (1992).

${ }^{41}$ V. Barone, L. Orlandini, and C. Adamo, J. Phys. Chem. 98, 13185 (1994).

${ }^{42}$ C. J. Umrigar, K. G. Wilson, and J. W. Wilkins, Phys. Rev. Lett. 60, 1719 (1988).

${ }^{43}$ M. S. Schuurman, W. D. Allen, and H. F. Schaefer, J. Comput. Chem. 26, 1106 (2005).

${ }^{44}$ J. L. Duncan and J. Harper, Mol. Phys. 51, 371 (1984).

${ }^{45}$ P. E. Cade and W. M. Huo, J. Chem. Phys. 47, 614 (1967).

${ }^{46}$ M. Bajdich, L. Mitas, G. Drobny, and L. K. Wagner, Phys. Rev. B 72, 75131 (2005).

${ }^{47}$ J. Kolorenc, H. Shuming, and L. Mitas, Phys. Rev. B 82, 115108 (2010).

${ }^{48}$ C. J. Umrigar and C. Filippi, Phys. Rev. Lett. 94, 150201 (2005).

${ }^{49}$ J. Toulouse and C. J. Umrigar, J. Chem. Phys. 126, 84102 (2007).

${ }^{50}$ C. J. Umrigar, J. Toulouse, C. Filippi, S. Sorella, and R. G. Hennig, Phys. Rev. Lett. 98, 110201 (2007).

${ }^{51}$ J. Toulouse and C. J. Umrigar, J. Chem. Phys. 128, 174101 (2008).

${ }^{52}$ B. Braïda, J. Toulouse, M. Caffarel, and C. J. Umrigar, J. Chem. Phys. 134, 084108 (2011).

${ }^{53}$ A. Le Floch, Mol. Phys. 72, 133 (1991).

${ }^{54}$ D. Feller, C. M. Boyle, and E. R. Davidson, J. Chem. Phys. 86, 3424 (1987).

${ }^{55}$ A. C. Venkatachar, R. C. Taylor, and R. L. Kuczkowski, J. Mol. Struct. 38, 17 (1977).

${ }^{56}$ K. Yagi, K. Hirao, T. Taketsugu, M. W. Schmidt, and M. S. Gordon, J. Chem. Phys. 121, 1383 (2004).

${ }^{57}$ K. K. Irikura, J. Phys. Chem. Ref. Data 36, 389 (2007).

${ }^{58}$ P. Meier, M. Neff, and G. Rauhut, J. Chem. Theory Comput. 7, 148 (2011).

${ }^{59}$ I. Ema, J. M. G. De la Vega, G. Ramirez, R. Lopez, J. F. Rico, H. Meissner, and J. Paldus, J. Comput. Chem. 24, 859 (2003).

${ }^{60}$ A. B. Burg, J. Am. Chem. Soc. 74, 3482 (1952).

${ }^{61}$ C. W. Bauschlicher and A. Ricca, Chem. Phys. Lett. 237, 14 (1995).

${ }^{62}$ C. H. DePuy, R. Gareyev, J. Hankin, G. E. Davico, M. Krempp, and R. Damrauer, J. Am. Chem. Soc. 120, 5086 (1998).

${ }^{63}$ X. Zeng and G. E. Davico, J. Phys. Chem. A 107, 11565 (2003). 